PROCEEDINGS OF THE

AMERICAN MATHEMATICAL SOCIETY

Volume 128, Number 3, Pages 933-937

$\mathrm{S}$ 0002-9939(99)05111-4

Article electronically published on July 28, 1999

\title{
ON BIACCESSIBLE POINTS IN THE JULIA SET OF A CREMER QUADRATIC POLYNOMIAL
}

\author{
DIERK SCHLEICHER AND SAEED ZAKERI
}

(Communicated by Michael Handel)

\begin{abstract}
We prove that the only possible biaccessible points in the Julia set of a Cremer quadratic polynomial are the Cremer fixed point and its preimages. This gives a partial answer to a question posed by C. McMullen on whether such a Julia set can contain any biaccessible point at all.
\end{abstract}

\section{INTRODUCTION}

Every quadratic polynomial in the complex plane with an irrationally indifferent fixed point is affinely conjugate to a unique quadratic polynomial of the form

$$
P: z \mapsto e^{2 \pi i \theta} z+z^{2},
$$

where $0<\theta<1$ is irrational and the indifferent fixed point is located at the origin. When $P$ is holomorphically linearizable about 0, we call it a Siegel polynomial. On the other hand, when the fixed point at the origin is nonlinearizable, $P$ is called a Cremer polynomial. By the theorem of Brjuno-Yoccoz [Y], $P$ is a Siegel polynomial if and only if $\theta$ satisfies the Brjuno condition:

$$
\sum_{n=1}^{\infty} \frac{\log q_{n+1}}{q_{n}}<+\infty
$$

where the $q_{n}$ appear as the denominators of the rational approximations coming from the continued fraction expansion of $\theta$.

Recall that the filled Julia set of $P$ is

$$
K(P)=\left\{z \in \mathbb{C}: \text { The orbit }\left\{P^{\circ n}(z)\right\}_{n \geq 0} \text { is bounded }\right\}
$$

and the Julia set of $P$ is the topological boundary of the filled Julia set:

$$
J(P)=\partial K(P) .
$$

Both sets are nonempty, compact, connected and the filled Julia set is full, i.e., the complement $\mathbb{C} \backslash K(P)$ is connected. Every connected component of the interior of $K(P)$ is a topological disk called a bounded Fatou component of $P$. According to Sullivan, every bounded Fatou component must eventually map to the immediate basin of attraction of an attracting periodic point, or to an attracting petal for a parabolic periodic point, or to a periodic Siegel disk for $P$ (see for example [Mi]). On the other hand, by [D] a polynomial of degree $d \geq 2$ can have at most $d-1$

Received by the editors February 19, 1998 and, in revised form, May 9, 1998.

1991 Mathematics Subject Classification. Primary 58F23; Secondary 30D40.

Key words and phrases. Julia set, Cremer point, biaccessible point, hedgehog. 
nonrepelling periodic orbits. It follows that in the Cremer case $K(P)$ has no interior, so that $K(P)=J(P)$.

\section{Definitions}

Given such a quadratic polynomial $P$, there exists a conformal isomorphism

$$
\varphi: \overline{\mathbb{C}} \backslash K(P) \rightarrow \overline{\mathbb{C}} \backslash \overline{\mathbb{D}}
$$

with $\varphi(\infty)=\infty$ and $\varphi^{\prime}(\infty)>0$, which conjugates $P$ to the squaring map:

$$
\varphi(P(z))=(\varphi(z))^{2} .
$$

The $\varphi$-preimages of the radial lines and circles centered at the origin are called the external rays and equipotentials of $K(P)$. The external ray $R_{t}$, by definition, is

$$
\varphi^{-1}\left\{r e^{2 \pi i t}: r>1\right\},
$$

where $t \in \mathbb{R} / \mathbb{Z}$ is called the angle of the ray. From (2) it follows that

$$
P\left(R_{t}\right)=R_{2 t}(\bmod 1) \text {. }
$$

We say that $R_{t}$ lands at $p \in J(P)$ if $\lim _{r \rightarrow 1} \varphi^{-1}\left(r e^{2 \pi i t}\right)=p$. A point $p \in J(P)$ is called accessible if there exists a simple arc in $\mathbb{C} \backslash K(P)$ which starts at infinity and terminates at $p$. According to a theorem of Lindelöf (see for example $[\mathrm{R}]$ ), $p$ is accessible exactly when there exists an external ray landing at $p$. We call $p$ biaccessible if it is accessible through at least two distinct external rays. By a theorem of $\mathrm{F}$. and M. Riesz [Mi], $K(P) \backslash\{p\}$ is disconnected whenever $p$ is biaccessible. It is interesting that the converse is also true. More precisely, if there are at least $n>1$ connected components of $K(P) \backslash\{p\}$, then at least $n$ distinct external rays land at $p$ (see for example $[\mathrm{Mc}]$ ).

\section{SOME KNOWN RESULTS}

Very little is known about the topology of the Julia set of $P$ in the Cremer case or the dynamics of $P$ on its Julia set. The following theorem summarizes the basic results in this direction:

Theorem 1. Let $P$ be a Cremer quadratic polynomial. Then

(a) The Julia set $J(P)$ cannot be locally connected [Su].

(b) Every neighborhood of the Cremer fixed point contains infinitely many repelling periodic orbits of $P[\mathrm{Y}]$.

(c) The critical point $c=-e^{2 \pi i \theta} / 2$ is recurrent, i.e., $c$ belongs to the closure of its orbit $\left\{P^{\circ n}(c)\right\}_{n>0}[\mathrm{Ma}]$.

(d) The critical point $c$ is not accessible from $\mathbb{C} \backslash J(P)[\mathrm{K}]$.

See also $[\mathrm{S} \varnothing]$ for the so-called "Douady non-landing theorem," which says that for a generic Cremer quadratic polynomial there is an external ray which accumulates on the Cremer fixed point and its preimage. Perez-Marco has shown that for every Cremer quadratic polynomial there exists an external ray whose prime-end impression contains the Cremer fixed point and its preimage [P2]. Both results shed some light on why the Julia set of a Cremer quadratic polynomial fails to be locally connected. 


\section{HedgeHogs}

Let $P$ be a Cremer quadratic polynomial. Let $U$ be a simply connected domain with compact closure which contains the indifferent fixed point 0 of $P$. Suppose that $P$ is univalent in a neighborhood of the closure $\bar{U}$. Then there exists a set $H=H_{U}$ with the following properties:

(i) $0 \in H \subset \bar{U} \cap J(P)$,

(ii) $H$ is compact, connected and full,

(iii) $H \cap \partial U$ is nonempty,

(iv) $P(H)=H$.

Such an $H$ is called a hedgehog for the restriction $\left.P\right|_{U}: U \rightarrow \mathbb{C}$. The existence of such completely invariant sets is proved by Perez-Marco [P1].

Hedgehogs turn out to be useful because of the following nice construction: Uniformize the complement $\mathbb{C} \backslash H$ by the Riemann map $\psi: \mathbb{C} \backslash H \rightarrow \mathbb{C} \backslash \overline{\mathbb{D}}$ and consider the induced map $g=\psi \circ P \circ \psi^{-1}$ which is defined (by (iv) above) and holomorphic in an open annulus $\{z \in \mathbb{C}: 1<|z|<r\}$. Use the Schwarz Reflection Principle to extend $g$ to the annulus $\left\{z \in \mathbb{C}: r^{-1}<|z|<r\right\}$. The restriction of $g$ to the unit circle $\mathbb{T}$ will then be a real-analytic diffeomorphism whose rotation number is exactly $\frac{1}{2 \pi i} \log P^{\prime}(0)=\theta \in \mathbb{R} / \mathbb{Z}$. This allows us to transfer results from the more developed theory of circle diffeomorphisms to the less explored theory of indifferent fixed points of holomorphic maps.

Using the above construction, it is not hard to prove the following fact (see [P2]):

Proposition 2. The only point in a hedgehog which can be biaccessible from outside of the hedgehog is the indifferent fixed point.

In fact, let $p$ be a biaccessible point in the hedgehog $H$ and $p \neq 0$. Then one can find a simple arc $\gamma$ in $\mathbb{C} \backslash H$ which starts and terminates at $p$ and does not encircle the indifferent fixed point 0 . Let $D$ be the bounded connected component of $\mathbb{C} \backslash(H \cup \gamma)$ and let $D^{\prime}=\psi(D)$. $D^{\prime}$ is bounded by the simple arc $\psi(\gamma)$ and an interval $I$ on the unit circle. Since $g$ has irrational rotation number on the unit circle $\mathbb{T}$, for some integer $N$ we have $\bigcup_{i=0}^{N} g^{\circ i}(I)=\mathbb{T}$. By choosing $\gamma$ close enough to $H$, we can assume that $g, g^{\circ 2}, \cdots, g^{\circ N}$ are all defined on $D^{\prime}$ and $\bigcup_{i=0}^{N} g^{\circ i}\left(D^{\prime}\right)$ contains an entire outer neighborhood of $\mathbb{T}$. It follows that $\bigcup_{i=0}^{N} P^{\circ i}(D)$ covers an entire deleted neighborhood of $H$. This is possible only if $0 \in \bar{D}$ which contradicts our assumption.

\section{THE THEOREM}

For the rest of this note, it will be more convenient to use the normal form

$$
P: z \mapsto z^{2}+v
$$

for a quadratic polynomial $P$ in (1), which can be achieved by an affine conjugation. In this normal form the origin is now the critical point, $P(0)=v$ is the critical value, and the two fixed points are located at

$$
\alpha, \beta=\frac{1}{2}(1 \pm \sqrt{1-4 v}) .
$$

The indifferent fixed point 0 in (1) is now located at the fixed point $\alpha$, which is the fixed point with smaller real part (note that $\alpha=\beta$ only when $v=1 / 4$ ). 
Since $P(z)=P(-z)$ by (3), the Julia set $J(P)$ is invariant under the $180^{\circ}$ rotation $\tau: z \mapsto-z$. If $U$ is an open Jordan domain in the plane such that $\bar{U} \cap \tau(\bar{U})=\emptyset$, it follows that $P$ is univalent in some Jordan domain $V$ containing the closure $\bar{U}$.

Now we can state the main theorem:

Theorem 3. Let $P: z \mapsto z^{2}+v$ be a Cremer quadratic polynomial with the indifferent fixed point $\alpha$. Let $z \in J(P)$ be biaccessible. Then there exists a nonnegative integer $k$ such that $P^{\circ k}(z)=\alpha$.

The proof begins as follows: Assume by way of contradiction that the forward orbit of $z$ never hits the fixed point $\alpha$. Let $R$ and $R^{\prime}$ be two distinct external rays that land at $z$. Consider the connected component $W$ of $\mathbb{C} \backslash\left(R \cup R^{\prime} \cup\{z\}\right)$ which does not contain $\alpha$. Since $\bigcup_{n \geq 0} P^{-n}(\alpha)$ is dense in $J(P)$ and $W$ intersects the Julia set, there exists a smallest positive integer $n$ such that $P^{-n}(\alpha) \cap W$ is nonempty. Let $\alpha_{-n}$ be a point in this intersection. Define

$$
R_{n-1}=P^{\circ n-1}(R), R_{n-1}^{\prime}=P^{\circ n-1}\left(R^{\prime}\right) .
$$

These rays are distinct because otherwise the forward orbit of $z$ would have to hit the critical point 0 which implies accessibility of 0 , and this contradicts Theorem $1(\mathrm{~d})$. Clearly both rays land at $z_{n-1}=P^{\circ{ }^{-1}}(z)$.

We claim that the ray pair $\left(R_{n-1}, R_{n-1}^{\prime}\right)$ separates the fixed point $\alpha$ from its preimage $-\alpha$. Assume it does not. Then the connected component $W_{n-1}$ of $\mathbb{C} \backslash\left(R_{n-1} \cup R_{n-1}^{\prime} \cup\left\{z_{n-1}\right\}\right)$ which contains the fixed point $\alpha$ also contains $-\alpha$. Consider $P^{\circ n-1}$ as a finite-degree branched covering $\hat{\mathbb{C}} \rightarrow \hat{\mathbb{C}}$ and let $\left\{O_{1}, \cdots, O_{m}\right\}$ be the collection of the connected components of $P^{-(n-1)}\left(W_{n-1}\right)$. Each $O_{i}$ is an unbounded simply connected domain whose boundary is formed by two or more external rays in $P^{-(n-1)}\left(R_{n-1} \cup R_{n-1}^{\prime}\right)$, and the mapping $P^{\circ n-1}: O_{i} \rightarrow W_{n-1}$ is proper holomorphic. In particular, since $W_{n-1}$ contains $\alpha$, it follows that each $O_{i}$ intersects $P^{-(n-1)}(\alpha)$. On the other hand, $P^{\circ n-1}\left(\alpha_{-n}\right)=-\alpha \in W_{n-1}$, which means $\alpha_{-n}$ belongs to $O_{i}$ for some $1 \leq i \leq m$. Since external rays do not cross, it follows that $O_{i} \subset W$. But this means that $W$ intersects $P^{-(n-1)}(\alpha)$, contradicting minimality of $n$.

Therefore, the ray pair $\left(R_{n-1}, R_{n-1}^{\prime}\right)$ separates the fixed point $\alpha$ from its preimage $-\alpha$. Without loss of generality, we can assume that these two rays actually separate $\alpha$ from the critical point 0 , for otherwise we could replace them by the symmetric pair $\left(\tau\left(R_{n-1}\right), \tau\left(R_{n-1}^{\prime}\right)\right)$. To simplify the notation, let $U=W_{n-1}$ be the connected component of $\mathbb{C} \backslash\left(R_{n-1} \cup R_{n-1}^{\prime} \cup\left\{z_{n-1}\right\}\right)$ which contains $\alpha$. To make $U$ have compact closure, one can cut it off by some equipotential curve (see Figure 1).

First we observe that $\left.P\right|_{\bar{U}}$ is univalent. In fact, if this were not true, $\bar{U}$ would have to intersect $\tau(\bar{U})$. Since both $\bar{U}$ and $\tau(\bar{U})$ are homeomorphic to the closed disk, this would imply that the boundary ray pairs $\left(R_{n-1}, R_{n-1}^{\prime}\right)$ and $\left(\tau\left(R_{n-1}\right), \tau\left(R_{n-1}^{\prime}\right)\right)$ intersect, which is impossible since distinct rays are disjoint (in case $R_{n-1}^{\prime}=\tau\left(R_{n-1}\right)$, the landing point would have to be the critical point which is ruled out by Theorem $1(\mathrm{~d}))$.

By the remark before the statement of the theorem, we can find a slightly larger Jordan domain $V \supset \bar{U}$ such that $\left.P\right|_{V}$ is still univalent. We consider a hedgehog $H_{V}$ for the restriction $\left.P\right|_{V}: V \rightarrow \mathbb{C}$ as in $\S 4$. By property (iii) of the definition of a hedgehog, $H_{V}$ has to reach the boundary of $V$. Since $H_{V}$ is connected and intersects $U$, it has to intersect the boundary of $U$ as well. But $H_{V} \subset J(P)$ and 


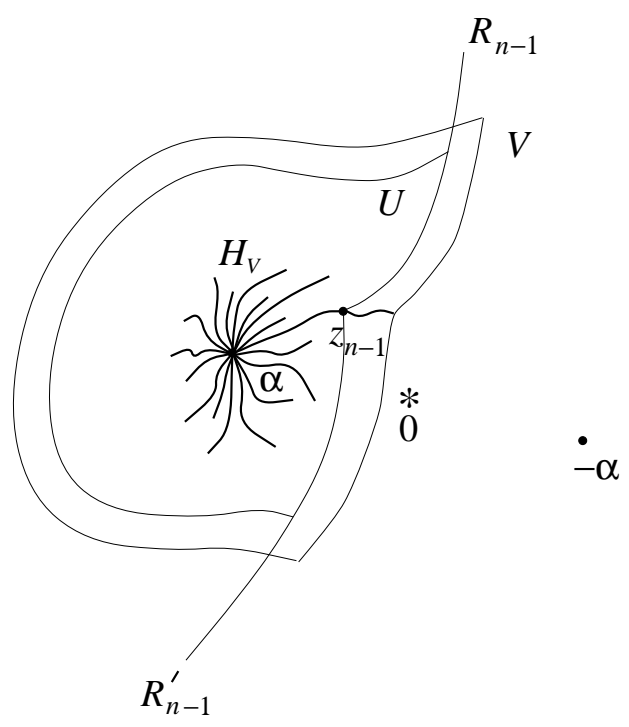

FiguRE 1

$\partial U \cap J(P)=\left\{z_{n-1}\right\}$. Hence $z_{n-1} \in H_{V}$. Since $z_{n-1}$ is biaccessible from outside of the Julia set, it follows that $H_{V} \backslash\left\{z_{n-1}\right\}$ is disconnected. Therefore, $z_{n-1}$ is biaccessible from outside of $H_{V}$. This contradicts Proposition 2, and finishes the proof of Theorem 3 .

\section{REFERENCES}

[D] A. Douady, Systemes dynamiques holomorphes, Asterisque, 105-106 (1983) 36-63. MR 85h:58090

[K] J. Kiwi, Non-accessible critical points of Cremer polynomials, SUNY at Stony Brook IMS preprint, 1996/2.

[Ma] R. Mañe, On a lemma of Fatou, Bol. Soc. Bras. Mat., 24 (1993) 1-12. MR 94g:58188

[Mc] C. McMullen, Complex Dynamics and Renormalization, Annals of Math Studies, vol. 135, 1994. MR 96b:58097

[Mi] J. Milnor, Dynamics in One Complex Variable: Introductory Lectures, SUNY at Stony Brook IMS preprint, 1990/5.

[P1] R. Perez-Marco, Fixed points and circle maps, Acta Math., 179 (1997) 243-294. MR 99a:58130

[P2] R. Perez-Marco, Topology of Julia sets and hedgehogs, Prépublications Math. Orsay, 1994.

[R] W. Rudin, Real and Complex Analysis, 3rd ed., McGraw-Hill, 1987. MR 88k:00002

[Sø] D. Sørensen, Accumulation theorems of quadratic polynomials, Ergod. Th. and Dynam. Sys., 6 (1996) 555-590. MR 97h:58135

[Su] D. Sullivan, Conformal Dynamical Systems, Springer Lecture Notes in Mathematics, 1007 (1983) 725-752. MR 85m:58112

[Y] J.C. Yoccoz, Petits Diviseurs en Dimension 1, Asterisque 231, 1995. MR 96m:58214

Fakultät für Mathematik, Technische Universität München, Barer Strasse 23, D-80290 München, Germany

E-mail address: dierk@mathematik.tu-muenchen.de

Department of Mathematics, SUNy at Stony Brook, New York 11794-3651

E-mail address: zakeri@math.sunysb.edu 